\title{
SELECTIVITY FOR CONSECUTIVE GAS-SOLID CATALYTIC REACTION*
}

\author{
RYozo TOEI, KAZUHIRo NAKANISHI** \\ AND MORIO OKAZAKI \\ Department of Chemical Engineering, Kyoto University, Kyoto
}

\begin{abstract}
The rate of consecutive hydrogenation of acetylene with nickel-on-kieselguhr catalysts was studied both experimentally and theoretically under such conditions that the effects of intraparticle diffusional resistance of micro and macro pores could be ignored, in order to examine the intrinsic reaction mechanism in detail, and the selectivity for a consecutive gas-solid catalytic reaction was investigated.

It was found that the rate steps on the catalytic surface, especially the desorption step of intermediate product and surface reaction step, played a predominant role in the selectivity.
\end{abstract}

\section{Introduction}

Selectivity in a consecutive reaction $(\mathrm{A} \rightarrow \mathrm{B} \rightarrow \mathrm{C})$ for porous catalysts has been the subject of a number of investigators, theoretical and experimental. But a lot of problems still remain unsolved, because there are so many complicated factors that govern selectivity, such as intraparticle diffusional resistance and physical or chemical properties of the catalytic surface.

The effect of the intraparticle diffusional resistance on selectivity was studied by Wheeler ${ }^{9}$, Carberry ${ }^{2}$ and recently by Komiyama and Inoue ${ }^{4,5}$. In particular, Komiyama and Inoue studied the rate of the hydrogenation of acetylene and pointed out that the diffusional resistance within micropores played an important role on the selectivity, but they paid little attention to the transport phenomena on the catalytic surface. On the other hand, chemists tend to study mainly from the view point of the physical or chemical characteristics of the surface and in most cases ignore the effects of the pore structure.

The purpose of the present study is to show both experimentally and theoretically the role of the catalytic surface in the selectivity, taking the effect of intraparticle diffusion into consideration. It was pointed out that in addition to the intraparticle diffusional process, the rate steps on the surface such as adsorption, surface reaction and desorption cannot be ignored when one considers the mechanism of reaction on heterogeneous catalysts.

* Received on August 10, 1973

Presented at the Autumn Meeting of The Soc. of Chem.

Engrs., Japan, at Osaka, November 1971

** Department of Food Science and Technology, Kyoto University, Kyoto

干606 京都市左京区北白川追分町

京都大学農学部食品工学科 中西一弘
We carried out the consecutive hydrogenation of acetylene with nickel-on-kieselguhr catalysts of which micropores were obliterated by annealing, in order to eliminate the effect of the diffusional resistance within micropores and to examine the surface reaction mechanism in detail.

It was observed that the value of the selectivity for the formation of ethylene was increased by diminishing the pellet size, but it never approached unity and it depended on the partial pressure of acetylene. The experimental results could be well explained by the combined surface reaction- and desorptioncontrolling mechanism without considering intraparticle diffusional steps.

\section{Selectivity of the Consecutive Reaction without considering Diffusional Steps}

\section{1 Reaction mechanism}

The reaction scheme for a consecutive hydrogenation of acetylene in gas phase is given by
A
(acetylene) (ethylene) (ethane)

and is considered to consist of the steps which are shown schematically in Fig. 1, where the step of intraparticle diffusion is neglected.

The complete steady-state mass balance equations for the reaction scheme are shown as follows, assuming that the mechanisms of adsorption and desorption

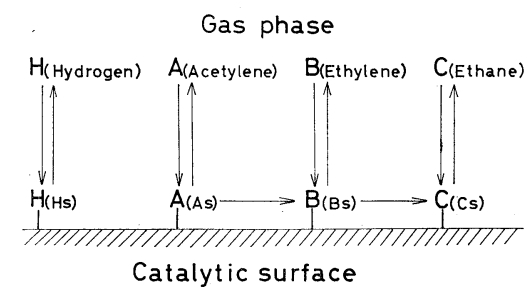

Fig. 1 Reaction mechanism 
follow the Langmuir monomolecular layer theory.

$$
\begin{aligned}
& \frac{d C_{A S}}{d t}=0=\left(k_{a}\right)_{A} p_{A} C_{V S}-\left(k_{d}\right)_{A} C_{A S}-r_{A S} \\
& \frac{d C_{B S}}{d t}=0=\left(k_{a}\right)_{B} p_{B} C_{V S}-\left(k_{d}\right)_{B} C_{B S}+r_{A S}-r_{B S} \\
& \frac{d C_{C S}}{d t}=0=\left(k_{a}\right)_{C} p_{C} C_{V S}-\left(k_{d}\right)_{C} C_{C S}+r_{B S} \\
& \frac{d C_{H S}}{d t}=0=\left(k_{a}\right)_{H} p_{H} C_{V S}-\left(k_{d}\right)_{H} C_{H S}-r_{A S}-r_{B S}
\end{aligned}
$$

where $k_{a}$ and $k_{d}$ represent adsorption and desorption rate constants; $C_{A S}, C_{B S}, C_{H S}$ and $C_{V S}$ represent surface concentrations of A (acetylene), B (ethylene), $\mathrm{C}$ (ethane), H (hydrogen) and vacant cataytic site; and $r_{A S}$ and $r_{B S}$ stand for surface reaction rates for $\mathrm{A}_{\mathrm{S}} \rightarrow \mathrm{B}_{\mathrm{S}}$ and $\mathrm{B}_{\mathrm{S}} \rightarrow \mathrm{C}_{\mathrm{S}}$ respectively.

Eq. (4) should be omitted in the case where adsorbed hydrogen does not participate in the reaction.

\section{2 Surface reaction rate forms for the hydrogenation of acetylene}

As the hydrogenation of acetylene involves two molecules, acetylene and hydrogen, two kinds of mechanism for the surface reaction can be considered:* the Langmuir-Hinshelwood model (L-H model) with both A (acetylene ) or B (ethylene) and $\mathrm{H}$ (hydrogen) reacting in the adsorbed state, and the Rideal model with $\mathrm{A}$ or $\mathrm{B}$ in the adsorbed state reacting with $\mathrm{H}$ in the gas phase.

For the $\mathrm{L}-\mathrm{H}$ model, $r_{A S}$ and $r_{B S}$ are given by

$$
\begin{aligned}
& r_{A S}=\left\{\left(k_{r}\right)_{A} / C_{T S}\right\} C_{A S} C_{H S} \\
& r_{B S}=\left\{\left(k_{r}\right)_{B} / C_{T S}\right\} C_{B S} C_{H S}
\end{aligned}
$$

where $C_{T S}$ is the concentration of total active sites on the surface.

For the Rideal model, we find

$$
\begin{aligned}
& r_{A S}=\left\{\left(k_{r}\right)_{A}^{\prime} / \pi\right\} C_{A S} p_{H} \\
& r_{B_{S}}=\left\{\left(k_{r}\right)_{B}^{\prime} / \pi\right\} C_{B S} p_{H}
\end{aligned}
$$

where $\left(k_{r}\right)_{A},\left(k_{r}\right)_{A}^{\prime}$ and $\left(k_{r}\right)_{B},\left(k_{r}\right)_{B}^{\prime}$ represent surface reaction rate constants for $A_{\mathrm{S}} \rightarrow B_{S}$ and $B_{S} \rightarrow C_{S}$ respectively; and $\pi$ is the total presssure.

\section{3 Assumptions for the analysis}

The assumptions for the analysis are summarized as follows.

(1) The diffusional step within pores is not considered.

(2) Both the desorption step of intermediate product (ethylene) and the surface reaction step are regarded as rate-limiting steps, and the adsorption and desorption rates of acetylene, ethane and hydrogen are

\footnotetext{
* Generally, in the hydrogenation of acetylene over metal catalysts, hydrogen is dissociatively adsorbed, and ethyl and vinyl radicals are observed on the catalytic surface.

In this paper, it is assumed that the reaction takes place in the molecular form.
}

sufficiently high, so that adsorption equilibria of A, $\mathrm{C}$ and $\mathrm{H}$ exist.

The physical meaning is that ethylene formed on the catalytic surface does not desorb immediately into the gas phase, but has some possibility to react further to ethane.

Generally, all the steps may contribute more or less to the overall reaction, but it is too complicated to take them all into consideration. This will be discussed in the latter part of this paper in detail.

(3) The reaction is irreversible.

(4) In the Rideal model, the amount of adsorbed hydrogen is regarded as negligibly small.

From the assumption (2), the surface concentrations of $\mathrm{A}, \mathrm{C}$, and $\mathrm{H}$ are expressed, using the adsorption equilibrium constants $K_{A}, K_{C}$ and $K_{H}$, as

$$
\begin{aligned}
& C_{A S}=K_{A} p_{A} C_{V S} \\
& C_{C S}=K_{C} p_{C} C_{V S} \\
& C_{H S}=K_{H} p_{H} C_{V S}
\end{aligned}
$$

where

$$
\begin{aligned}
& K_{A}=\left(k_{a}\right)_{A} /\left(k_{d}\right)_{A} \\
& K_{C}=\left(k_{a}\right)_{C} /\left(k_{d}\right)_{C} \\
& K_{H}=\left(k_{a}\right)_{H} /\left(k_{d}\right)_{H}
\end{aligned}
$$

And the concentration of total active sites should be constant as

$$
C_{T S}=C_{A S}+C_{B S}+C_{C S}+C_{H S}+C_{V S}
$$

\section{4 Mathematical formulations}

The mass balance equation for $C_{B S}$ (Eq. (2)) can be solved analytically using Eqs. (5) (6) and Eqs. (9) (15) for the L-H model and Eqs. (7) (15) for the Rideal model, respectively. Accordingly, the expressions for the reaction rate and selectivity can be easily obtained as shown in the following section.

1.4.1 The case of the L-H model Eqs. (2) and (15) are rearranged, using Eqs. (5) (6) and (9) (14) as

$$
\begin{aligned}
\left(k_{a}\right)_{B} C_{V S} p_{B}- & \left(k_{d}\right)_{B} C_{B S}+\left\{\left(k_{r}\right)_{A} / C_{T S}\right\} K_{A} p_{A} K_{H} p_{H} C_{V S}{ }^{2} \\
- & -\left\{\left(k_{r}\right)_{B} / C_{T S}\right\} C_{B S} K_{H} p_{H} C_{V S}=0
\end{aligned}
$$

and

$$
\begin{aligned}
C_{T S}= & K_{A} p_{A} C_{V S}+C_{B S}+K_{C} p_{C} C_{V S} \\
& +K_{H} p_{H} C_{V S}+C_{V S}
\end{aligned}
$$

From Eqs. (16) and (17), $C_{V S}$ can be obtained as

$$
C_{V S}=\frac{-B+\sqrt{B^{2}+4 A C}}{2 A}
$$

where

$$
\begin{aligned}
A= & \left(k_{r}\right)_{A} K_{A} p_{A} K_{H} p_{H}+\left(k_{r}\right)_{B} K_{H} p_{H}\left(K_{A} p_{A}+K_{C} p_{C}\right. \\
& \left.+K_{H} p_{H}+1\right) \\
B= & \left(k_{d}\right)_{B} C_{T S}\left(K_{A} p_{A}+K_{B} p_{B}+K_{C} p_{C}+K_{H} p_{H}+1\right) \\
& -\left(k_{r}\right)_{B} C_{T S} K_{H} p_{H} \\
C= & \left(k_{d}\right)_{B} C_{T S}
\end{aligned}
$$

The rate of formation of gaseous A and B can be 
written as

$$
\begin{aligned}
d p_{A} / d t= & -\left\{\left(k_{r}\right)_{A} / C_{T S}\right\} C_{A S} C_{H S} \\
= & -\left\{\left(k_{r}\right)_{A} / C_{T S}\right\} K_{A} p_{A} K_{H} p_{H} C_{V S}{ }^{2} \\
d p_{B} / d t= & \left(k_{d}\right)_{B}\left(C_{B S}-K_{B} p_{B} C_{V S}\right) \\
= & \left(k_{d}\right)_{B}\left\{C_{T S}-\left(K_{A} p_{A}+K_{B} p_{B}+K_{C} p_{C}\right.\right. \\
& \left.\left.+K_{H} p_{H}+1\right) C_{V S}\right\}
\end{aligned}
$$

Substituting Eq. (18) into Eqs. (22) and (23) to obtain

$$
\begin{aligned}
d p_{A} / d t= & -\left\{\left(k_{r}\right)_{A} / C_{T S}\right\} K_{A} p_{A} K_{H} p_{H} \\
& \times\left(\frac{-B+\sqrt{B^{2}+4 A C}}{2 A}\right)^{2} \\
d p_{B} / d t= & \left(k_{d}\right)_{B}\left\{C_{T S}-\left(K_{A} p_{A}+K_{B} p_{B}+K_{C} p_{C}\right.\right. \\
& \left.\left.+K_{H} p_{H}+1\right) \frac{-B+\sqrt{B^{2}+4 A C}}{2 A}\right\}
\end{aligned}
$$

Dividing Eq. (25), by Eq. (24), we obtain the expression for the selectivity as

$$
\begin{aligned}
S= & -\frac{d p_{B} / d t}{d p_{A} / d t} \\
= & {\left[( k _ { d } ) _ { B } C _ { T S } \left\{C_{T S}-\left(K_{A} p_{A}+K_{B} p_{B}+K_{C} p_{C}+K_{H} p_{H}+1\right)\right.\right.} \\
& \left.\left.\times \frac{\left.\left.-B+\sqrt{B^{2}+4 A C}\right\}\right]}{2 A}\right\}\right] \\
& \times \frac{1}{\left(k_{r}\right)_{A} K_{A} p_{A} K_{H} p_{H}\left\{\left(-B+\sqrt{\left.\left.B^{2}+4 A C\right) / 2 A\right\}^{2}}\right.\right.}
\end{aligned}
$$

The expressions for the intrinsic rate of formation of gaseous $R_{A 0}$ and the intrinsic selectivity $S_{0}$ can be obtained by setting $p_{B}=p_{C}=0$ in Eqs. (24) and (26).

$$
\begin{gathered}
R_{A 0}=-\left(k_{r}\right)_{A} C_{T S} K_{A} p_{A} K_{H} p_{H} \\
\times\left(\frac{-B^{\prime}+\sqrt{B^{\prime 2}+4 \gamma A^{\prime}}}{2 A^{\prime}}\right)^{2} \\
S_{0}=\frac{2 \gamma}{S} A^{\prime} \\
\times \frac{2 A^{\prime}-\left(K_{A} p_{A}+K_{H} p_{H}+1\right)\left(-B^{\prime}+\sqrt{B^{\prime 2}+4 \gamma A^{\prime}}\right)}{K_{A} p_{A} K_{H} p_{H}\left(-B^{\prime}+\sqrt{B^{\prime 2}+4 \gamma A^{\prime}}\right)^{2}}
\end{gathered}
$$

where

$$
\begin{gathered}
A^{\prime}=s K_{A} p_{A} K_{H} p_{H}+K_{H} p_{H}\left(K_{A} p_{A}+K_{H} p_{H}+1\right) \\
B^{\prime}=\gamma\left(K_{A} p_{A}+K_{H} p_{H}+1\right)-K_{H} p_{H} \\
\gamma=\left(k_{d}\right)_{B} /\left(k_{r}\right)_{B} \\
s=\left(k_{r}\right)_{A} /\left(k_{r}\right)_{B}
\end{gathered}
$$

1.4.2 The case of the Rideal model In the same way as described above, $C_{V S}$ is derived, combining Eqs. (2), (7) (10), (12) (13) and (15) as

$$
\begin{aligned}
C_{V S}=\left\{\left(k_{r}\right)_{B}^{\prime} p_{H}+\left(k_{d}\right)_{B}\right\} C_{T S} /\left[\left(k_{r}\right)_{A}^{\prime} K_{A} p_{A} p_{H}+\left(k_{d}\right)_{B} K_{B} p_{B}\right. \\
\left.+\left(K_{A} p_{A}+K_{C} p_{C}+1\right)\left\{\left(k_{r}\right)_{B}^{\prime} p_{H}+\left(k_{d}\right)_{B}\right\}\right]
\end{aligned}
$$

and the intrinsic reaction rate of formation of gaseous $\mathrm{A}$ and the intrinsic selectivity are expressed as

$$
R_{A 0}=\frac{\left(k_{r}\right)_{A}^{\prime} K_{A} p_{A}\left(p_{H}+\gamma^{\prime}\right) C_{T S} p_{H}}{s^{\prime} K_{A} p_{A} p_{H}+\left(K_{A} p_{A}+1\right)\left(p_{H}+\gamma^{\prime}\right)}
$$

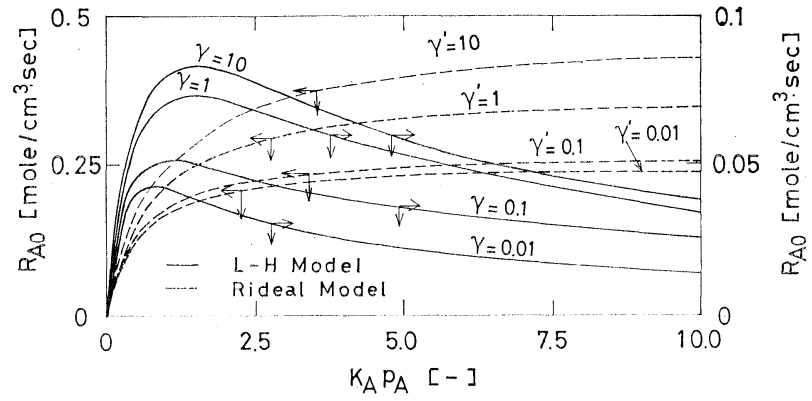

Fig. 2 Reaction rate curves for the combined surface reaction- and desorption-limiting mechanism

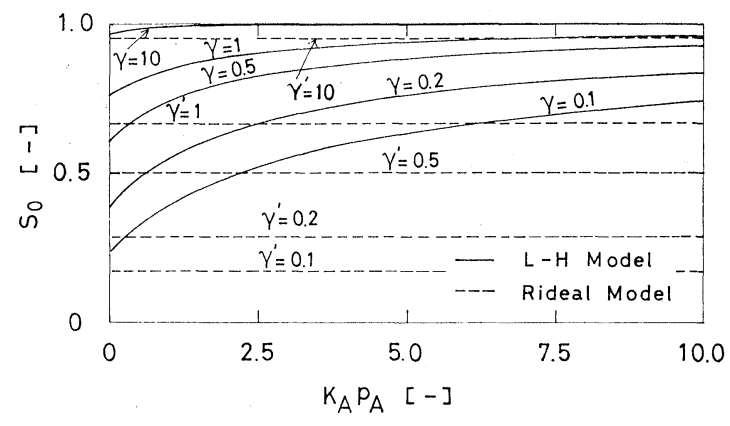

Fig. 3 Selectivity curves for the combined surface reaction- and desorption-limiting mechanism

$$
S_{0}=\frac{1}{1+p_{H} / \gamma^{\prime}}
$$

where

$$
\begin{aligned}
\gamma^{\prime} & =\left(k_{d}\right)_{B} /\left(k_{r}\right)_{B}^{\prime} \\
s^{\prime} & =\left(k_{r}\right)_{A}^{\prime} /\left(k_{r}\right)_{B}^{\prime}
\end{aligned}
$$

Figure 2 shows the intrinsic rate as function of $K_{A} p_{A}$ for various values of $\gamma$ or $\gamma^{\prime}$ in which solid lines show the calculated results by the L-H model (Eq. (27)) and dotted lines imply those by the Rideal model (Eq. (34)). In the calculation, the values of $\left(k_{r}\right)_{A} C_{T S}$ or $\left(k_{r}\right)_{A}^{\prime} C_{T S}, s$ or $s^{\prime}$ and $K_{H} p_{H}$ are specified as $1.0,1.0$ and 0.5 , respectively. As seen from the figure, all the curves have the same shape regardless of the values of $\gamma$ or $\gamma^{\prime}$. In other words, by inspection alone, one cannot tell whether the intrinsic rate curves result from the combined surface reaction- and desorptionlimiting mechanism ( $\gamma$ or $\gamma^{\prime}$ is small) or form the pure surface reaction-limiting mechanism $\left(\gamma\right.$ or $\gamma^{\prime}$ is large).

In the case of the $\mathrm{L}-\mathrm{H}$ model, the reaction rate increases rapidly to a maximum value and then decreases gradually as the partial pressure of gas A increases. On the other hand, in the case of the Rideal model the rate increases asymptotically to a maximum value.

In Fig. 3, the intrinsic selectivity $S_{0}$ is plotted as function of $K_{A} p_{A}$ for various values of $\gamma$ or $\gamma^{\prime}$ for both models according to Eqs. (28) and (35), respectively. The values of $S_{0}$ appreciably depend on the values of $\gamma$ and in the case of the $\mathrm{L}-\mathrm{H}$ model they are also affected by the values of $p_{A}$. In the case of the 


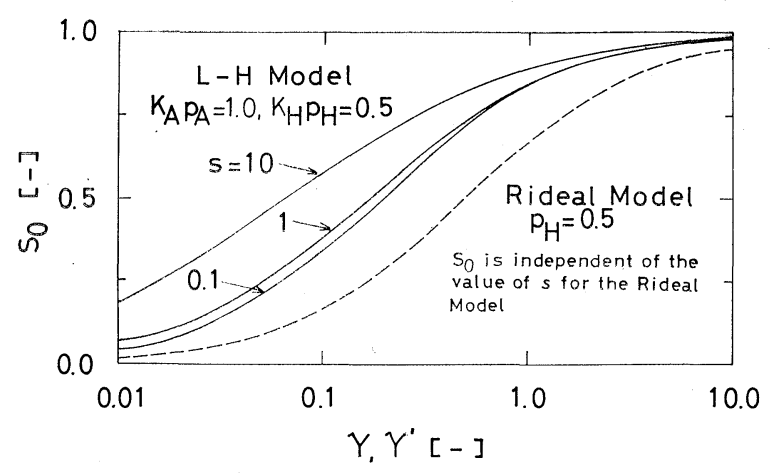

Fig. $4 S_{0}$ vs. $\gamma, \gamma^{\prime}$

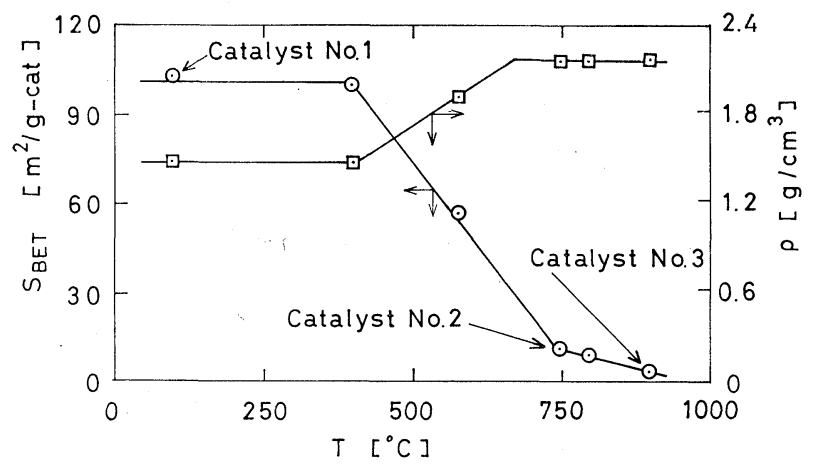

Fig. 5 The BET surface area and bulk density vs. annealing temperature

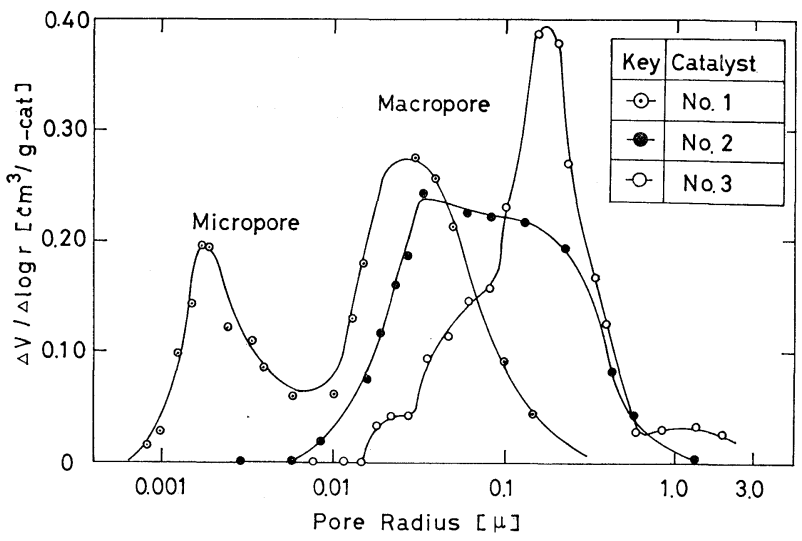

Fig. 6 Pore distribution curves

Rideal model, they remain constant regardless of the values of $K_{A} p_{A}$ for the fixed values of $\gamma^{\prime}$.

In Fig. 4, the intrinsic selectivity is shown as a function of $s$ or $s^{\prime}$ in which the effect of $\gamma$ or $\gamma^{\prime}$ can be clearly observed. $S_{0}$ does not depend on the value of $s^{\prime}$ for the Rideal model.

\section{Catalyst}

The catalyst used in this study is a nickel-onkieselguhr catalyst (supplied from Nikki Chemicals) which is the same as that used in the previous paper ${ }^{8)}$. As described in detail in that paper, the catalyst has a so-called "micro-macro pore structure". It is composed of micropores which are the spaces among the aggregates of fine nickel crystals and macropores of the catalyst support. The support (kieselguhr) is much more thermostable than nickel crystals, so it is

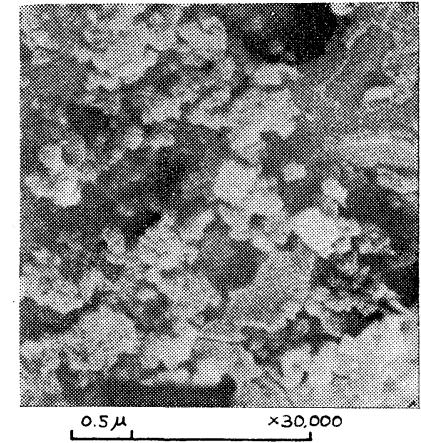

Photo. 1 Figure of the fractured surface of the Catalyst No. 1

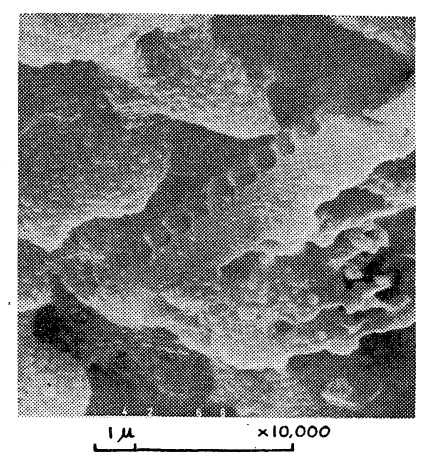

Photo. 2 Figure of the fractured surface of the Catalyst No. 3

anticipated that micropores are obliterated with annealing.

Original nickel-on-kieselguhr catalysts were annealed before reaction at the five temperature levels 400 , $580,750,800$ and $900^{\circ} \mathrm{C}$ under hydrogen stream for four hours. In Fig. 5, the BET surface area and bulk density are plotted against the annealing temperature. The BET area diminishes to less than one-tenth that of the original catalyst at temperatures higher than $750^{\circ} \mathrm{C}$, and this may imply the vanishing of micropores.

In Fig. 6, the pore distribution curves of the original catalyst (hereafter called Catalyst No. 1), the catalyst annealed at $750^{\circ} \mathrm{C}$ (Catalyst No. 2) and that at $900^{\circ} \mathrm{C}$ (Catalyst No. 3) are shown. As seen from the figure, micropores of Catalyst Nos. 2 and 3 are regarded as having vanished.

To confirm the vanishing of micropores, photographs of the catalysts were taken by a scanning electron microscope. Photo. 1 shows the fractured surface of Catalyst No. 1. Photo. 2 shows that of Catalyst No. 3. Comparing these two photographs, it is found that the aggregates of nickel crystals melt into spherical shape and disperse on the support under the treatment of annealing. Moreover, it was found that no chemical combination between nickel and kieselguhr occured during the process of annealing. This was confirmed by the fact that most of the nickel contained in the catalyst dissolved when it 
was dipped in dilute solution of hydrochloric acid.

\section{Experiments and Results}

\section{1 Procedure}

A flow sheet of the reaction apparatus is shown in Fig. 7 and is similar to that used in the previous paper $^{8)}$. The reactor was made of a stainless tube of $10 \mathrm{~mm}$ I.D. and was heated by an electric heater. The temperature of the center of the catalyst bed was controlled within $\pm 0.5^{\circ} \mathrm{C}$ by a P.I. temperature controller.

Two copper-constantan thermocouples were put in the catalyst bed, one of them near the center of the catalyst bed and the other near the wall of the reactor. The temperature difference between them was within $2^{\circ} \mathrm{C}$ during the experiment. No appreciable temperature difference along the longitudinal direction of the bed was detected.

Preactivation of the catalysts was performed in a hydrogen stream for two hours at $180^{\circ} \mathrm{C}$. Thereafter, the catalyst bed was cooled to the reaction temperature. Mixtures of nitrogen and acetylene gases were fed at a constant flow rate.

At the initial stage of the reaction the activity of the catalyst was quite high, but after about one hour from the start of reaction the catalyst activity became completely constant. At this moment the outlet gas was analyzed by gas chromatography.

The reaction was carried out under such conditions that hydrogen gas was in large excess compared with acetylene gas in order to prevent the deactivation of the catalyst during the reaction. And the total flow rate was so high that the diffusional resistance through gas film could be ignored. The reacting conditions are summarized in Table $\mathbf{1}$.

\section{2 The dependency of the selectivity on the con-} version of acetylene

By analyzing the composition of product gas, the conversion of acetylene and the selectivity, defined as the ratio of the amount of ethylene to that of acetylene reacted, were obtained in each experiment. The feed gas always has the same composition $\left(\mathrm{C}_{2} \mathrm{H}_{2}=\right.$ $0.043 \mathrm{~atm}, \mathrm{H}_{2}=0.57 \mathrm{~atm}$ ), so that the observed selectivity depends only on the catalyst size and the conversion of acetylene.

The observed selectivity, $S$, and the conversion, $x$, are plotted in Figs. 8 and 9 for several differentsize catalysts. Figure 8 shows the experimental results obtained with Catalyst No. 1, and Fig. 9 illustrates those obtained with Catalyst No. 3.

In each case, the selectivity is improved by decrease of catalyst size, and at particle diameters of $0.1 \mathrm{~mm}$ and $0.3 \mathrm{~mm}$ the value of selectivity is independent of size for Catalysts No. 1 and No. 3 respectively. These values can be regarded as imply-

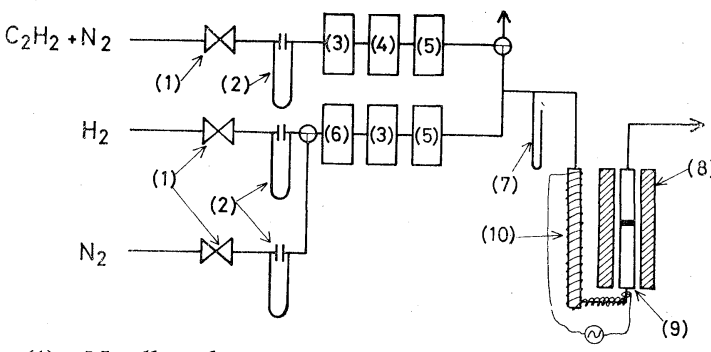

(1) Needle valve

(2) Capillary flow meter

(3) Activated charcoal tube

(4) Soda-lime tube

(5) Silica-gel tube

(7) Manometer

(8) Electric heater

(9) Reactor

(10) Preheater

(6) Oxygen removal tube (Pd catalysts)

Fig. 7 Reaction apparatus

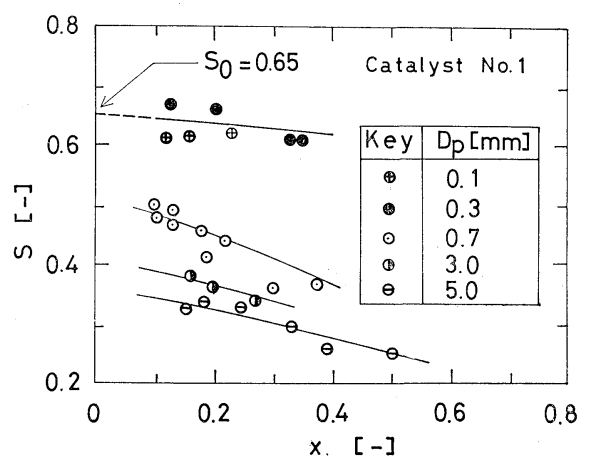

Fig. 8 Selectivity vs. conversion (Catalyst No. 1)

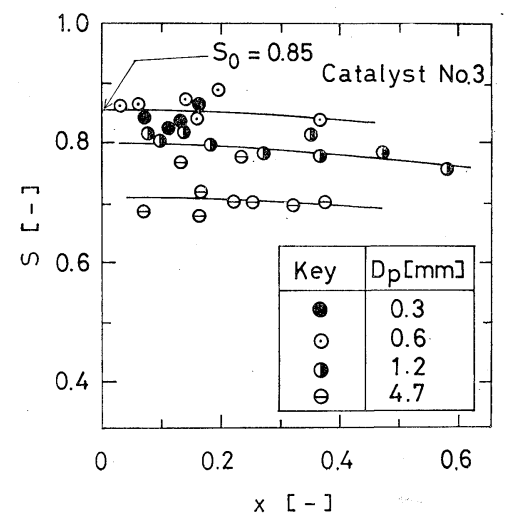

Fig. 9 Selectivity vs. conversion (Catalyst No. 3)

\begin{tabular}{|c|c|}
\hline & Reacting conditions \\
\hline Catalysts & $\begin{array}{l}\text { Original nickel-on-kieselguhr catalyst (Catalyst } \\
\text { No. 1), the catalyst annealed at } 900^{\circ} \mathrm{C} \text { (Catalyst } \\
\text { No. 3) }\end{array}$ \\
\hline \multicolumn{2}{|l|}{ Catalyst size } \\
\hline Catalyst No. 1 & $\begin{array}{l}5^{\phi} \times 5^{H} \mathrm{~mm} \text { cylindrical pellet and crushed } \\
\text { particles, } D_{p}=3.0,0.7,0.3,0.1 \mathrm{~mm}\end{array}$ \\
\hline Catalyst No. 3 & $\begin{array}{l}4.7^{\phi} \times 4.7^{H} \mathrm{~mm} \text { cylindrical pellet and crushed } \\
\text { particles, } D_{p}=1.2,0.6,0.3 \mathrm{~mm}\end{array}$ \\
\hline Catalyst weight & $0.08-2.3 \mathrm{~g}$ \\
\hline \multirow[t]{3}{*}{ Feed gases } & $0.043 \mathrm{~atm}$ \\
\hline & $0.570 \mathrm{~atm}$ \\
\hline & $\mathrm{N}_{2} \quad 0.387 \mathrm{~atm}$ \\
\hline \multicolumn{2}{|c|}{ Total flow rate $40-120 \mathrm{l} / \mathrm{hr}\left(20^{\circ} \mathrm{C}, 1 \mathrm{~atm}\right)$} \\
\hline \multicolumn{2}{|c|}{ Reaction temperature $\quad 123 \pm 2^{\circ} \mathrm{C}$} \\
\hline Conversion & $0.034-0.582$ \\
\hline
\end{tabular}

ing those where there is no macropore diffusional resistance. 


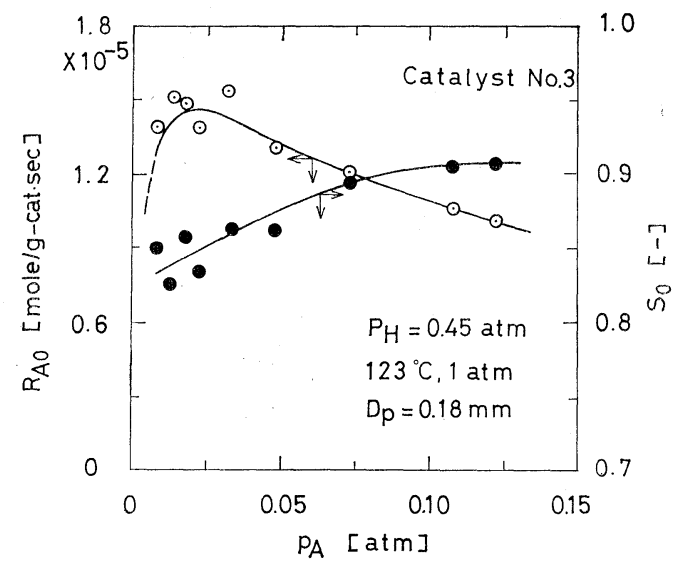

Fig. 10 Intrinsic rate and selectivity vs. $p_{A}$ (experiments)

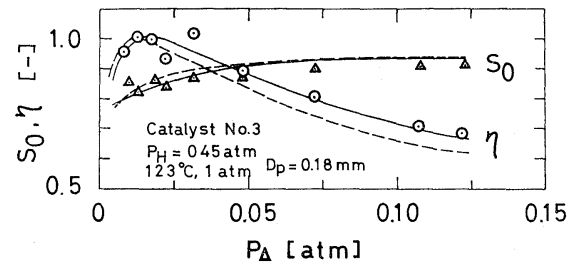

Fig. 11 Comparison between the experimental results and calculated ones

However, even in such a limiting condition the intrinsic selectivity, $S_{0}$, defined as the value at the acetylene conversion of zero, does not reach unity but remains at 0.65 and 0.85 for Catalysts No. 1 and No. 3 respectively. The value obtained with Catalyst No. 2 was also 0.85 .

As the reason for the improvement of the intrinsic selectivity, the following two factors are considered. (1) The diffusional resistance within micropores is originally large but then disappears, and (2) the catalytic surface is changed by the process of annealing. But it is not clear within the limits of the present investigations about which factors are predominant. Further study from the viewpoint of the reaction rate forms is required.

\section{3 The dependency of the reaction rate on the partial pressure of acetylene for Catalyst No. 3}

The Catalyst No. 3 was used for the rate measurement. The size of the catalyst was $0.3 \mathrm{~mm}$, for which the diffusional resistance not only within micropores also within macropores can be ignored. In Fig. 10, $R_{A 0}$ and $S_{0}$ are plotted against the partial pressure of acetylene at the fixed value of $p_{H}$, i.e., 0.45 atm. As seen from the figure, $R_{A 0}$ takes a maximum value near $p_{A}=0.01 \mathrm{~atm}$ and then decreases gradually, and $S_{0}$ increases. These two curves resemble those calculated by the $\mathrm{L}-\mathrm{H}$ model for the reaction scheme as indicated in Figs. 2 and 3, respectively.

\footnotetext{
* As a matter of fact, the value of $K_{H} / K_{A}$ is very small as determined in Section 4.1 , so that $K_{H} p_{H} / K_{A} p_{A}$ takes a relatively small value as long as the value of $p_{H} / p_{A}$ is not so large.
}

\section{Analysis and Discussions}

\section{1 Estimation of rate constants}

In the preceding section, it was shown that the relations of the reaction rate and selectivity to the partial pressure of acetylene could be well explained qualitatively by Eqs. (27) and (28). Here, a quantitative treatment is made.

Four unknown parameters appearing in Eqs. (27) and (28), i.e., $K_{A}, K_{H}, s$ and $\gamma$, are determined as 179.97, $3.91,10.0$ and 1.0 respectively, using the non-linear least squares method proposed by Marquardt ${ }^{6)}$ so as to make the deviations from the experimental data minimum.

In Fig. 11, the calculated results (dotted lines) are compared with the experimental data, in which $\eta$ shows the relative value of $R_{A 0}$, i.e., the value of $R_{A 0}$ divided by its maximum value $\left(1.5 \times 10^{-5} \mathrm{~mol} / \mathrm{g}\right.$-cat . sec). The agreement between the experiments and calculated results is considered to be a reasonably good one, considering the experimental errors and the difficulty of determining strictly the four unknown parameters from two kinds of available data.

In the figure, the solid lines show the calculated lines obtained with the values of $0.0436,8.65$ and 1.74 for $K_{H} / K_{A}, s$ and $\gamma$ respectively, in which it is assumed that $K_{A} p_{A}+K_{H} p_{H}$ is much larger than 1 so that the problem of determining four unknown parameters is reduced to that of determining three. Better agreement between experiment and theory is found.

\section{2 Approximate form for intrinsic selectivity}

From Eqs. (22) and (23), $S_{0}$ is expressed as

$$
S_{0}=\frac{\left(k_{d}\right)_{B} C_{B S}}{\left\{\left(k_{r}\right)_{A} / C_{T S}\right\} C_{A S} C_{H S}}
$$

and

$$
\begin{aligned}
\frac{1}{S_{0}}-1 & =\frac{\left\{\left(k_{r}\right)_{A} / C_{T S}\right\} C_{A S} C_{H S}-\left(k_{d}\right)_{B} C_{B S}}{\left(k_{d}\right)_{B} C_{B S}} \\
& =\frac{\left\{\left(k_{r}\right)_{B} / C_{T S}\right\} C_{B S} C_{H S}}{\left(k_{d}\right)_{B} C_{B S}} \\
& =\frac{C_{H S}}{\gamma C_{T S}}
\end{aligned}
$$

$C_{H S}$ is written using Eqs. (11) and (18) as

$$
\begin{aligned}
C_{H S} & =K_{H} p_{H} C_{V S} \\
& =K_{H} p_{H} \frac{-B+\sqrt{B^{2}+4 A C}}{2 A}
\end{aligned}
$$

where $B^{2}$ is larger than $4 A C$, Eq. (40) becomes

$$
\begin{aligned}
C_{H S} & =K_{H} p_{H} \frac{C}{B} \\
& =\frac{K_{H} p_{H}\left(k_{d}\right)_{B} C_{T S}}{\left(k_{d}\right)_{B}\left(K_{A} p_{A}+K_{H} p_{H}+1\right)-\left(k_{r}\right)_{B} K_{H} p_{H}}
\end{aligned}
$$

Accordingly, Eq. (39) is rearranged, assuming that $K_{H} p_{H} / K_{A} p_{A}$ is relatively small*, as 
Table 2 Rate-limiting steps

\begin{tabular}{|c|c|c|c|c|}
\hline \multirow[t]{2}{*}{ Rate-limiting steps } & \multicolumn{2}{|l|}{ L-H model } & \multicolumn{2}{|c|}{ Rideal model } \\
\hline & $R_{A 0}$ & $S_{0}$ & $R_{A 0}$ & $S_{0}$ \\
\hline $\begin{array}{l}\text { Combined surface reaction-and adsorption } \\
\text { (of A)-limiting mechanism }\end{array}$ & $\begin{array}{l}\left(k_{r}\right)_{A} C_{T S \xi}\left\{1-\left(K_{A} p_{A}+K_{H} p_{H}+1\right)\right. \\
\quad \times\left(-D+\sqrt{\left.D^{2}-4 \xi E\right)} / 2 E\right\}\end{array}$ & 1 & $\frac{\left(k_{r}\right)_{A}{ }^{\prime} C_{T S} K_{A} p_{A} p_{H}}{K_{A} p_{A}+1+p_{H} / \xi^{\prime}}$ & 1 \\
\hline $\begin{array}{l}\text { Combined surface reaction-and desorption } \\
\text { (of C)-limiting mechanism (pure surface } \\
\text { reaction-limiting mechanism) }\end{array}$ & $\frac{\left(k_{r}\right)_{A} C_{T S} K_{A} p_{A} K_{H} p_{H}}{\left(K_{A} p_{A}+K_{H} p_{H}+1\right)^{2}}$ & 1 & $\frac{\left(k_{r}\right)_{A}^{\prime} C_{T S} K_{A} p_{A} p_{H}}{K_{A} p_{A}+1}$ & 1 \\
\hline $\begin{array}{l}\text { Combined surface reaction-and desorption } \\
\text { (of intermediate product)-limiting mechanism }\end{array}$ & Eq. (27) & Eq. (28) & Eq. (34) & Eq. (35) \\
\hline where & $\begin{array}{l}D=K_{H} p_{H}(\xi-1)+\xi\left(K_{A} p_{A}+1\right) \\
E=K_{H} p_{H}\left(K_{H} p_{H}+1\right)\end{array}$ & & $\begin{array}{l}\left(k_{d}\right)_{A} /\left(k_{r}\right)_{A} \\
\left(k_{d}\right)_{A} /\left(k_{r}\right)_{A}{ }^{\prime}\end{array}$ & \\
\hline
\end{tabular}

$$
\begin{aligned}
\frac{1}{S_{0}}-1 & =\frac{K_{H} p_{H}}{\gamma\left(K_{A} p_{A}+K_{H} p_{H}+1\right)-K_{H} p_{H}} \\
& =\frac{K_{H} p_{H} / K_{A} p_{A}}{\gamma+(\gamma-1) K_{H} p_{H} / K_{A} p_{A}+\gamma / K_{A} p_{A}} \\
& \cong \alpha \frac{p_{H}}{p_{A}}+\beta
\end{aligned}
$$

In Fig. 12, the experimental values of intrinsic selectivity are plotted against $p_{H} / p_{A}$ in which the reported data, mainly by Bond ${ }^{11}$, are also shown.

As seen from the figure, Eq. (42) fits all the data fairly well except ours. In our experiment, the values of $p_{H} / p_{A}$ extend over a wide range, i.e., from 3.75 to 45.0, and $S_{0}$ deviates from Eq. (42) at large values of $p_{H} / p_{A}$.

\section{3 Validity of the Assumption (2)}

As described above, the experimental results obtained in this study can be well explained not only qualitatively but also quantitatively by the combined surface reaction-and desorption-limiting mechanism, using the Langmuir-Hinshelwood rate forms as the surface reaction rate equation.

In addition to this mechanism, two more combinations of rate-limiting steps can be considered, that is (a) the combined adsorption (of acetylene) and surface reaction mechanism and (b) the combined desorption (of ethane) and surface reaction mechanism. The step of the surface reaction cannot be omitted from the rate-limiting steps, because the reaction under consideration is irreversible. The analytical solutions for these cases are summarized in Table 2. As seen from the table, $S_{0}$ never depends on $p_{A}, p_{H}, \gamma$, or $s$ and takes a constant value, i.e., 1 , which contradicts the experimental results.

Generally, all the steps appearing in Fig. 1 may more or less contribute to the intrinsic reaction rate and selectivity, but taking them all into consideration, is too complicated and results in losing the physical meaning. It should be noted that the most important factor which affects the selectivity is the behavior of

\footnotetext{
* When the value of $p_{H} / p_{A}$ approaches zero, $S_{0}$ may take a value less than unity, because in such a limiting case $r$ may be affected by $p_{A}$ and $p_{H} . \quad \beta$ is a correction factor for this effect.
}

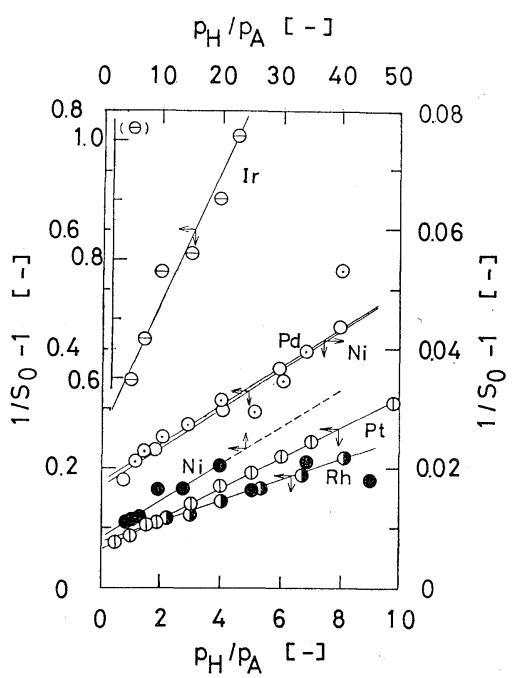

\begin{tabular}{clrrrl} 
Key & \multicolumn{1}{c}{ Catalyst } & $\begin{array}{c}\text { Reaction } \\
\text { temperature }\end{array}$ & $\alpha$ & $\beta$ & Literature \\
$\odot$ & Pd-alumina & $0^{\circ} \mathrm{C}$ & 0.0033 & 0.0175 & Bond \\
- & Ni-kieselguhr & $125^{\circ} \mathrm{C}$ & 0.0058 & 0.09 & This work \\
$\odot$ & $\mathrm{Rh}$-alumina & $50^{\circ} \mathrm{C}$ & 0.0175 & 0.08 & Bond $^{11}$ \\
$\odot$ & Pt-alumina & $110^{\circ} \mathrm{C}$ & 0.0245 & 0.06 & Bond $^{11}$ \\
$\odot$ & Ni-pumice & $80^{\circ} \mathrm{C}$ & 0.033 & 0.165 & Sheridan $^{7 \prime}$ \\
$\ominus$ & Ir-alumina & $130^{\circ} \mathrm{C}$ & 0.11 & 0.52 & Bond $^{11}$
\end{tabular}

Fig. $121 / S_{0}-1$ vs. $p_{H} / p_{A}$

intermediate product, but the steps of adsorption of reactants and the desorption of final product are not so important. As long as the discussion is limited to the intrinsic selectivity, it can be said at present that the Assumption (2) is reasonable. Detailed study of the behavior of individual steps is required in order to draw a complete conclusion.

\section{4 Comparison with other treatments}

Only a few studies have been reported on the problem of selectivity both experimentally and theoretically. Bond ${ }^{11}$ observed the dependency of $S_{0}$ on the partial pressure of hydrogen and explained it by a simple model, but little attention was paid to the effects of pore structure, and rate data were not observed. Moreover, the dependency of $S_{0}$ on the partial pressure of acetylene cannot be explained by that model.

Komiyama and Inoue ${ }^{4,5)}$ also studied the rate of hydrogenation of acetylene and observed 0.82 for $S_{0}$ 
$\left(p_{H} / p_{A}=2.0\right)$. They reasoned the fact that $S_{0}$ never approached unity, by the diffusional resistance of micropores, but they ignored the effect of the rate steps on the catalytic surface.

De Boer and van der Borg ${ }^{3)}$ theoretically analyzed the effects of the rate steps on the surface upon the selectivity for a simple reaction system but they did not prove it experimentally.

This paper shows both experimentally and theoretically the role in selectivity of the rate steps on the surface. The treatment must be applied to the other systems of consecutive reaction, such as the hydrogenation of butadiene, oxidation of ethylene and so on.

\section{Conclusions}

(1) The observed selectivity of intermediate product never approached unity even where the diffusional resistance of both micropores and macropores can be ignored. Through these experimental results, it was pointed out that the desorption step of intermediate product from the catalytic surface cannot be ignored, compared with the surface reaction step. (2) The dependency of the intrinsic selectivity and reaction rate on the partial pressure of acetylene was well explained both qualitatively and quantitatively by the surface reaction- and desorption-limiting mechanism with the Langmuir-Hinshelwood rate forms. (3) The intrinsic selectivity values observed by other investigators were well correlated by the simple relation $1 / S_{0}-1=\alpha\left(p_{H} / p_{A}\right)+\beta$ deduced from our model. (4) The intrinsic selectivity increased under the process of annealing the catalyst. But the predominant factor is not clear, and is the subject for a future study.

\section{Acknowledgment}

The authors wish to express their thanks to the Science Research Foundation of the Ministry of Education, Japan, for its financial support (Grant No. 8730).

They also would like to thank Seitetsu Kagaku Co., Ltd. for supplying acetylene gas and Nippon Shokubai Kagaku Co., Ltd. for providing facilities to use a scanning electron microscope, and Mr. Yasumi Shiozaki for his assistance in the experimental work.
}

\section{Nomenclature}

C $=$ concentration on the catalytic surface

\begin{tabular}{|c|c|}
\hline$D_{p}$ & $=$ particle diameter \\
\hline$K$ & $=$ adsorption equilibrium constant \\
\hline$k_{a}$ & $=$ adsorption rate constant \\
\hline$k_{d}$ & $=$ desorption rate constant \\
\hline$k_{r}, k_{r}^{\prime}$ & $=$ surface reaction rate constant \\
\hline$p$ & $=$ partial pressure \\
\hline$R$ & $=$ reaction rate \\
\hline$r$ & $=$ pore radius \\
\hline$r_{A S}$ & $=$ surface reaction rate for $A_{S} \rightarrow B_{S}$ \\
\hline$r_{B S}$ & $=$ surface reaction rate for $B_{S} \rightarrow C_{S}$ \\
\hline$S$ & $=$ selectivity \\
\hline$s, s^{\prime}$ & $\begin{aligned}= & \text { ratio of the value of }\left(k_{r}\right)_{A},\left(k_{r}\right)_{A}^{\prime} \text { to that of } \\
& \left(k_{r}\right)_{B},\left(k_{r}\right)_{B}^{\prime}\end{aligned}$ \\
\hline$T$ & $=$ temperature \\
\hline$V$ & $=$ pore volume \\
\hline$\alpha$ & $=$ constant appearing in Eq. (38) \\
\hline$\beta$ & $=$ constant appearing in Eq. (38) \\
\hline$\gamma, \gamma^{\prime}$ & $\begin{aligned}= & \text { ratio of the value of }\left(k_{r}\right)_{B},\left(k_{r}\right)_{B}^{\prime} \text { to that of } \\
& \left(k_{d}\right)_{B}\end{aligned}$ \\
\hline$\xi, \xi^{\prime}$ & $\begin{aligned}= & \text { ratio of the value of }\left(k_{r}\right)_{A},\left(k_{r}\right)_{A}^{\prime} \text { to that of } \\
& \left(k_{d}\right)_{A}\end{aligned}$ \\
\hline$\eta$ & $=$ relative value of $R_{A 0}$ to the maximum value $[-]$ \\
\hline$\rho$ & $=$ bulk density $\quad\left[\mathrm{g} / \mathrm{cm}^{3}\right]$ \\
\hline$\pi$ & $=$ total pressure \\
\hline
\end{tabular}

$\langle$ Subscripts〉

$\begin{array}{ll}A & =\text { gas component A (acetylene) } \\ B & =\text { gas component B (ethylene) } \\ C & =\text { gas component C (ethane) } \\ H & =\text { gas component } \mathrm{H} \text { (hydrogen) } \\ S & =\text { catalytic surface } \\ T & =\text { total active sites } \\ V & =\text { vacant active sites } \\ 0 & =\text { intrinsic value }\end{array}$

\section{Literature Cited}

1) Bond, G. C. and P. B. Wells: Proc. Int. Congr. Catalysis, 1, 1135, 1139, Paris (1961)

2) Carberry, J. J.: Chem. Eng. Sci., 17, 675 (1962)

3) de Boer, J. H. and van der Borg, R. J. A. M.: Proc. Intern. Congr. Catalysts, 1, 919, Paris (1961)

4) Komiyama, H. and H. Inoue: J. Chem. Eng. Japan, 1, 142 (1968)

5) Komiyama, H. and H. Inoue: J. Chem. Eng. Japan, 3, 206 (1970)

6) Marquardt, D. W.: J. Soc. Indust. Appl. Math., 2, 431 (1963)

7) Sheridan, J.: J. Chem. Soc., 373 (1944)

8) Toei, R. M. Okazaki, K. Nakanishi, Y. Kondo, M. Hayashi and Y. Shiozaki: J. Chem. Eng. Japan, 6, 50 (1973)

9) Wheeler, A.: "Catalysis" (Edited by Emmett P. H.), vol. II, Reinhold, New York (1955) 